\title{
Publizistenpreis der deutschen Bibliotheken 2020 geht an den freien Journalisten Johannes Nichelmann
}

https://doi.org/10.1515/bd-2020-0054

Der mit 7.500 Euro dotierte Publizistenpreis der deutschen Bibliotheken (HelmutSontag-Preis) geht in diesem Jahr an den freien Journalisten Johannes Nichelmann. Ausgezeichnet wird seine Reportage „Obdach Stadtbibliothek“, die in der Sendung Mikrokosmos des Deutschlandfunks erstmals am 1. Juni 2018 gesendet wurde.

In der Jurybegründung heißt es:

„Johannes Nichelmann möchte den Bibliotheken auf den Grund gehen: In seiner Reportage ,Obdach Stadtbibliothek' begibt er sich in den Alltag der Hamburger Bücherhalle am Hühnerposten und spricht mit ganz unterschiedlichen Besucher^innen und Mitarbeiter ${ }^{\star}$ innen. Da ist zum Beispiel Wolfgang, der seinen Job verloren hat und teilweise auf der Straße lebt. Oder Anneliese, die sich in der Bibliothek regelmäßig mit einer jungen Frau aus Afghanistan trifft und sie beim Deutschlernen unterstützt. Im anschließenden Gespräch diskutiert Nichelmann seine Eindrücke mit Simon Fregin, Jugendsozialarbeiter in der Stadtbibliothek am Mailänder Platz in Stuttgart, und Jonas Fansa von der Berliner Zentral- und Landesbibliothek, und fragt, wie sich Bibliotheken in Zeiten der Digitalisierung und gesellschaftlichen Veränderungen entwickeln.

Für die Jury zeichnet sich Nichelmanns Arbeit insbesondere dadurch aus, dass er seinen Protagonistinnen sehr nahe kommt und Fragen stellt, die für Bibliotheken relevant sind, sich aber nicht allein auf Bibliotheken beziehen: Welche Bedeutung haben öffentliche Räume für eine Stadtgesellschaft und wie müssen diese gestaltet sein, damit sie für die Besucher`innen auch soziale Orte sein können. Durch die Mischung aus O-Tönen der Besucher`innen und Mitarbeiter^innen, dem Interviewgespräch sowie seiner eigenen Eindrücke vermittelt Johannes Nichelmann unterschiedliche Perspektiven der Arbeit und Herausforderungen von Bibliotheken und bietet ein einfühlsames Abbild der Stadtgesellschaft, in der Bibliotheken viel mehr als Orte für Bücher sind.“

Der Beitrag „Obdach Stadtbibliothek“ ist nachzuhören auf der Webseite von Deutschlandfunk. ${ }^{1}$

1 https://www.deutschlandfunk.de/oeffentliche-kulturarbeit-obdach-stadtbibliothek.3381. de.html?dram:article_id=440098 [Zugriff: 26.03.2020]. 
Autor und Moderator: Johannes Nichelmann

Redaktion der Sendung: Marietta Schwarz

Erstausstrahlung: 1. Juni 2018

\section{Zum Preisträger}

Johannes Nichelmann, geboren 1989 in Berlin, studierte Politikwissenschaft an der Freien Universität Berlin. Seit 2008 arbeitet er als freier Reporter, Autor und Moderator für den öffentlich-rechtlichen Rundfunk. Zunächst für die rbb-Jugendwelle „Fritz“, später vor allem für Deutschlandfunk und Deutschlandfunk Kultur sowie zahlreiche ARD-Anstalten, ZDF und ARTE. Im Ullstein-Verlag erschien im September 2019 sein Sachbuch „Nachwendekinder - Die DDR, unsere Eltern und das große Schweigen“.

Ein Foto des Preisträgers Johannes Nichelmann kann hier ${ }^{2}$ in hoher Auflösung heruntergeladen werden.

\section{Preisverleihung}

Der Publizistenpreis der deutschen Bibliotheken wird gemeinsam vom Deutschen Bibliotheksverband (dbv), dem Berufsverband Bibliothek Information e.V. (BIB) und dem Verein Deutscher Bibliothekarinnen und Bibliothekare e.V. (VDB) verliehen. Über die Auswahl des Preisträgers entschied eine unabhängige Jury, bestehend aus Vertreter`innen der bibliothekarischen Verbände (BIB, dbv und VDB) sowie von Medien und Verlagen. Die Preisverleihung findet nach derzeitigem Stand am 29. Mai 2020 im Rahmen der Abschlussveranstaltung des Bibliothekartages in Hannover statt.

\section{Publizistenpreis der deutschen Bibliotheken}

Der Preis würdigt Publizisten, die das Bibliothekswesen durch herausragende Einzelbeiträge oder durch die Kontinuität sachgerechter Berichterstattung wirkungsvoll gefördert haben. Er wird seit 1987 jährlich verliehen. Die Initiative zur

2 https://www.bibliotheksverband.de/dbv/presse/pressefotos/preistraeger-publizistenpreis2020.html [Zugriff: 26.03.2020]. 
Preisverleihung geht auf die Anregung des ehemaligen dbv-Vorsitzenden Helmut Sontag (1934-1988) zurück. Von 2010 bis 2017 erfolgte die Ausschreibung gemeinsam mit der Wissenschaftlichen Buchgesellschaft (WBG). Seit 2018 schreiben die bibliothekarischen Verbände Deutscher Bibliotheksverband e.V. (dbv), Berufsverband Bibliothek Information e.V. (BIB), und der Verein Deutscher Bibliothekarinnen und Bibliothekare e.V. (VDB) den Publizistenpreis der deutschen Bibliotheken gemeinsam aus.

Weitere Informationen zum Publizistenpreis und zu den bisherigen Preisträger^innen gibt es hier ${ }^{3}$.

\section{Der Deutsche Bibliotheksverband e.V. (dbv)}

Der Deutsche Bibliotheksverband e.V. (dbv) vertritt mit seinen mehr als 2.100 Mitgliedern bundesweit rund 10.000 Bibliotheken mit 25.000 Beschäftigten und 11 Mio. Nutzer^innen. Sein zentrales Anliegen ist es, Bibliotheken zu stärken, damit sie allen Bürger*innen freien Zugang zu Informationen ermöglichen. Der Verband setzt sich ein für die Entwicklung innovativer Bibliotheksleistungen für Wissenschaft und Gesellschaft. Als politische Interessensvertretung unterstützt der dbv die Bibliotheken insbesondere auf den Feldern Informationskompetenz und Medienbildung, Leseförderung und bei der Ermöglichung kultureller und gesellschaftlicher Teilhabe für alle Bürgerinnen.

www.bibliotheksverband.de

\section{Pressekontakt}

Kristin Bäßler, Leitung Kommunikation / Pressesprecherin Tel.: 030 / 6449899 25, E-Mail: presse@bibliotheksverband.de URL: www.bibliotheksverband.de

$3 \mathrm{https} / / /$ www.bibliotheksverband.de/dbv/auszeichnungen/publizistenpreis/preistraeger.html [Zugriff: 26.03.2020]. 